Research Article

\title{
Modulation of Glucose Takeup by Glucose Transport on the Isolated OHCs
}

\author{
Xiao-ting Cheng, ${ }^{1,2}$ Feng-bo Yang, ${ }^{1,3}$ Qing-qing Jiang, ${ }^{1}$ Rong Zhang, ${ }^{2}$ Shi-ming Yang, ${ }^{1}$ \\ and Ning $Y \mathbf{u}$ iD 1 \\ ${ }^{1}$ Department of Otorhinolaryngology Head and Neck Surgery and Institute of Otorhinolaryngology, The PLA General Hospital, \\ Beijing 100853, China \\ ${ }^{2}$ Department of Otorhinolaryngology Head and Neck Surgery, The First Affiliated Hospital of Fujian Medical University, \\ Fujian 350005, China \\ ${ }^{3}$ Department of Otolaryngology, Affiliated Hospital of North Sichuan Medical College, Nanchong 637100, China
}

Correspondence should be addressed to Ning Yu; yuning12@sina.com

Received 21 January 2018; Accepted 3 March 2018; Published 5 April 2018

Academic Editor: Renjie Chai

Copyright (c) 2018 Xiao-ting Cheng et al. This is an open access article distributed under the Creative Commons Attribution License, which permits unrestricted use, distribution, and reproduction in any medium, provided the original work is properly cited.

Glucose is a fundamental source of energy for mammalian cells; however, whether glucose is taken up through the lateral walls of cochlear outer hair cells (OHCs) is unknown. The OHC lateral wall is complex, composed of a plasma membrane, cortical lattice, and subsurface cisternae. This study assessed the uptake of glucose by OHCs using live-cell microscopy and examined the distribution of glucose transporter isoforms by immunohistochemistry. We found that glucose transporter- 4 was mostly expressed on the lateral wall of OHCs. Glucose crossed the lateral walls of OHCs via glucose transporters- 4 mainly, and this process could be modulated. These results suggest that the lateral walls are involved in modulating energy transport into OHCs.

\section{Introduction}

The length of cochlear OHCs can change at a very high frequency when they receive acoustic signals $[1,2]$. Although electromotility is not directly dependent on ATP or other chemical intermediates $[3,4]$, these rapid changes in body length must consume a great deal of energy. The complex lateral cortex of an OHC is composed of three distinct layers, namely, the subsurface cisternae (SSC), cortical cytoskeletal lattice (CL), and plasma membrane (PM) [5]. The SSC is a specialized and substantial fraction of the endoplasmic membrane within OHCs; in guinea pigs, as many as twelve ordered layers line the lateral cytoplasmic surface of the PM [6]. The cortical lattice is an unusual protein skeleton associated with the outermost surface of the lateral cisternae beneath the PM. The outermost PM contains a high density of integral membrane proteins. One of these, prestin, a specialized electromotor protein, is expressed on the outer PM [7]. The relationship between glucose transporters and prestin is unclear $[8,9]$.
The functions of OHC lateral walls are understudied. How glucose is transported across the three membrane structures, whether they participate in energy transport, and whether glucose transporters are related to electromotility are unknown. Here, we assessed the cytoplasmic uptake of glucose by OHCs using live-cell microscopy and examined the subcellular localization of glucose transporter (GLUT) isoforms by immunohistochemistry. We observed that glucose crossed the membrane via glucose transporters and that this process could be controlled; further, we found that GLU4 was expressed on the subcellular localization. These results imply that the lateral walls of cochlear OHCs are involved in controlling energy transport.

\section{Materials and Methods}

2.1. Animal Preparation and Cochlear Cell Isolation. Cochlear cells were freshly isolated from adult guinea pigs $(250-300 \mathrm{~g})[10,11]$. Briefly, guinea pigs were anesthetized 
with an overdose of chloral hydrate $(0.3 \mathrm{ml} / 100 \mathrm{~g})$, and otic capsules were removed after decapitation. The otic capsules were dissected, and the isolated cochleas were put in normal extracellular solution (142 mM NaCl, $5.37 \mathrm{mM} \mathrm{KCl}, 1.47 \mathrm{mM}$ $\mathrm{MgCl}_{2}, 2 \mathrm{mM} \mathrm{CaCl} \cdot 2 \mathrm{H}_{2} \mathrm{O}$, and $10 \mathrm{mM}$ HEPES; $300 \mathrm{mOsm}$; $\mathrm{pH}$ 7.2). After removal of the bone and stria vascularis, the sensory epithelium (organ of Corti) was collected using a sharpened needle and further dissociated with trypsin $(0.5 \mathrm{mg} / \mathrm{ml})$ for $2-3 \mathrm{~min}$ with shaking. Cochlear cells were then transferred to a recording dish. All experimental procedures were performed at room temperature $\left(23^{\circ} \mathrm{C}\right)$. The animals were purchased from Vital River Laboratory Animal Technology Co. Ltd. (Beijing, China). All animal experiments were approved by the Institutional Animal Care and Use Committee of the Chinese PLA General Hospital (Beijing, China).

2.2. Experimental Agents. Adenosine $5^{\prime}$-triphosphate (ATP) disodium salt solution (cat. no. A6559-25UMO), indinavir (cat. no. Y-0000788), and WZB-117 (SML0621-5MG) were purchased from Sigma (St. Louis, MO). Antibodies against 4-[[[[4-(1,1-dimethylethyl)phenyl] sulfonyl] amino]methyl]N-3-pyridinylbenzamide (STF-31; cat. no. SC-364692) and prestin (H-294; cat. no. sc-30163) were purchased from Santa Cruz Biotechnology (Santa Cruz, CA). The dyes di-8ANEPPS (D3167), Hoechst 33342 (R37605), and 2-NBDG (N13195) were purchased from Molecular Probes (Eugene, OR). Antibodies against GLUT-1 (ab652) and GLUT-4 (ab654) were purchased from LifeSpan BioSciences Inc. (Seattle, WA). A perfusion system was used to apply reagents to cells. The animals were anesthetized for all procedures with an intraperitoneal injection of chloral hydrate.

2.3. Immunohistochemistry. Dissociated cochlear cells were fixed with $4 \%$ paraformaldehyde for $30 \mathrm{~min}$. After three washes with $0.01 \mathrm{M}$ PBS, the cells were incubated in blocking solution ( $10 \%$ goat serum in PBS with $0.25 \%$ Triton X-100) for $30 \mathrm{~min}$. The cells were then incubated with anti-GLUT or anti-prestin antibodies $(1: 100-250)$ in blocking solution at room temperature $\left(23^{\circ} \mathrm{C}\right)$ for $2 \mathrm{~h}$. In control experiments, the primary antibody was omitted. After three washes with PBS, the cells were incubated with Alexa Fluor 488-conjugated donkey anti-rabbit IgG (1:200; cat. no. A21206, Molecular Probes) or Alexa Fluor 568-conjugated donkey anti-goat IgG (1 :200; cat. no. A11057, Molecular Probes) in blocking solution at room temperature for $1 \mathrm{~h}$. For costaining with di8-ANEPPS to visualize the PM and cytoplasmic membranous organelles, the cells were further incubated in $30 \mu \mathrm{M}$ di-8-ANEPPS (D-3167; Molecular Probes) for $20 \mathrm{~min}$ after secondary antibody incubation. After completely washing out the dye with $0.01 \mathrm{M}$ PBS, staining was observed under a confocal microscope.

Cell nuclei were stained with Hoechst 33342 (R37605; Molecular Probes). Following incubation with the secondary antibody, pieces of dissociated cells were incubated with a dilution of Hoechst 33342 stock solution (2 drops per ml) at room temperature for 15-30 min and washed three times with PBS.
2.4. Live-Cell Imaging, Image Processing, and Statistical Analysis. Live-cell imaging was performed using an Applied Precision DeltaVision microscope (GE Healthcare, Issaquah, WA), and data were acquired and processed with the accompanying software. Fluorescence intensity was measured using ImageJ. All data are expressed as the mean \pm standard deviation (SD). Data were analyzed using an unpaired $t$-test (Student's $t$-test) for two groups and a one-way ANOVA with Bonferroni post hoc tests for multiple comparisons, as noted in the figure legends (SPSS 13.0 software; SPSS Inc., Chicago, IL). Differences with $p<0.05$ were deemed statistically significant. Error bars in the figures represent the SD.

\section{Results}

3.1. 2-NBDG Is Transported into the Cytoplasm of OHCs. 2NBDG is a novel fluorescent derivative of glucose that is useful for assessing glucose uptake activity [12]. A good viability of isolated OHCs can be sustained for two hours in normal extracellular solution [13], and the procedure for acquiring isolated $\mathrm{OHCs}$ in this experiment only need 15 minutes. As shown in Figure 1, following $20 \mathrm{~min}$ of exposure to 2-NBDG $(1 \mathrm{mM})$, the OHCs from the basal and apical turn displayed intracellular fluorescence, indicating uptake (Figures 1(b) and 1(d)). Control OHCs without perfusion with $2-\mathrm{NBDG}$ had minimal cellular fluorescence, indicating relatively little autofluorescence (data was not shown here). Hence, the OHCs appeared to take up 2-NBDG rapidly and retain it on the lateral wall and within the cytoplasm (Figures 1(b) and 1(d)).

Then we examined the concentration and time dependency of 2-NBDG uptake by OHCs. We perfused a series of 2-NBDG concentrations $(0.1 \mathrm{~mm} / 1,0.5 \mathrm{~mm} / \mathrm{l}, 1 \mathrm{~mm} / \mathrm{l}$, and $2 \mathrm{~mm} / \mathrm{l}$ ) to the OHCs that were isolated from all turns of the cochlea. The normalized fluorescence intensity (NFI) on the lateral wall and cytoplasm at each concentration was divided by the initial concentration $(0.1 \mathrm{~mm} / \mathrm{l})$ (Figure $1(\mathrm{e}))$. The NFI increased with the increase of 2-NBDG concentration. Similarly, fluorescence was normalized by dividing the value by that at time zero. The uptake of 2-NBDG increased gradually over time until reaching a plateau (Figure 1(f)). The results suggested that 2-NBDG uptake by $\mathrm{OHCs}$ depended on the concentration and time.

3.2. GLUT-1 and GLUT-4 Expression in OHCs. Despite the above results showing that 2-NBDG is taken up by OHCs, little is known about the expression and localization of glucose transporters in these cells. Previous studies have shown GLUT-1, GLUT-3, GLUT-4, GLUT-5, GLUT-8, GLUT-10, and GLUT-12 expressions in both the stria vascularis and the spiral ligament [14]. We examined the relative distribution of GLUT isoforms in OHCs by immunohistochemistry (Figure 2; some data was not shown). Staining for all glucose transporters except GLUT-4 was punctate and mostly close to the edge of the cell; GLUT-4 was distributed evenly along the lateral cell edges, similar to previous observations of prestin distribution [7]. We also observed the expression of transporters on the cuticular plate, which has not been previously reported and is consistent with our observation of 2-NBDG 


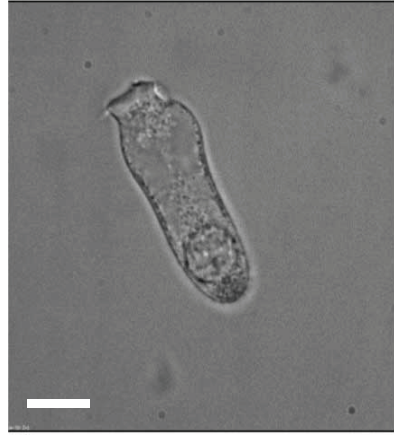

(a)

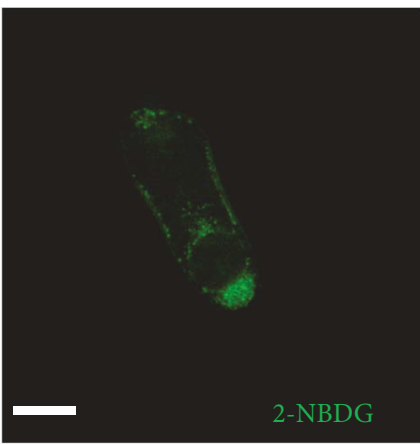

(b)

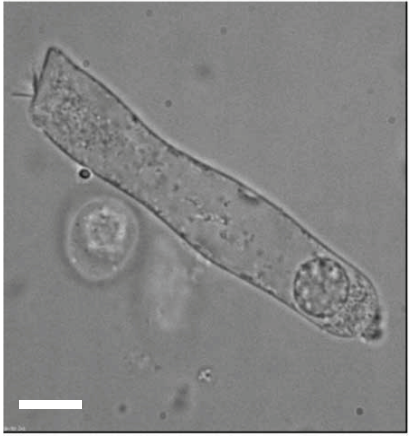

(c)

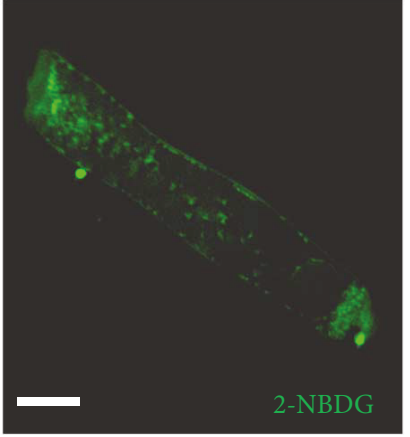

(d)

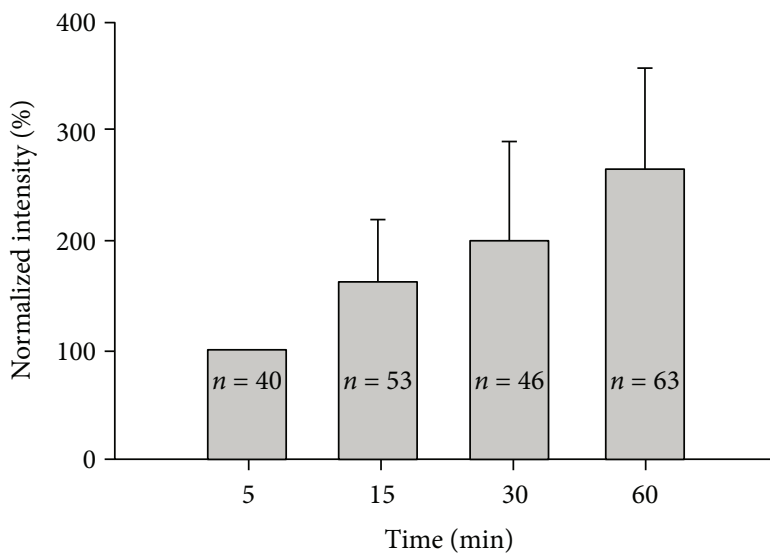

(f)

(e)

FIgURE 1: Uptake of 2-NBDG into OHCs and concentration or time-dependent uptake of 2-NBDG by OHCs. (a and c) Nomarski images. (b and d) Guinea pig OHCs of various lengths following exposure to 2-NBDG ( $1 \mathrm{mM}$ for 20 min; cells were imaged after a 10 min washout; green, 2-NBDG). (e) Normalized fluorescence intensity of 2-NBDG at varying concentrations. The concentration was measured at $20 \mathrm{~min}$ after giving the 2-NBDG. (f) Normalized fluorescence intensity of 2-NBDG over time. The concentration of 2-NBDG used was $1 \mathrm{mM} / \mathrm{l}$. Scale bars, $10 \mu \mathrm{m}$.

uptake by OHCs through the cuticular plate using live-cell imaging (data not shown). While previous reports indicated that OHCs did not express GLUT-5 [9], we observed that the outer hair cells expressed GLUT-5 scatteredly (data was not shown). These findings strongly suggest that a number of GLUT isoforms participate in glucose transport in OHCs.

3.3. OHC Uptake of 2-NBDG Is Modulated by GLUT-1 and GLUT-4 Antagonists. We next examined the effect of glucose antagonists on the uptake of 2-NBDG (Figure 3; all data are relative to untreated cells). STF-31 and WZB117 are GLUT1 antagonists $[15,16]$ and decreased the uptake of 2-NBDG to $0.9308 \pm 0.0909$ and $0.9561 \pm 0.1095$, respectively, though the reductions were small. Indinavir $(50 \mu \mathrm{mol} / \mathrm{l})[17,18]$ is a GLUT-4 antagonist and decreased the uptake of 2-NBDG to $0.5907 \pm 0.0649$.

\subsection{Comparison Dye or Antibody Staining Localization} between GLUT-4 and Di-8-ANEPPS. In addition to the lateral walls, some GLUT-4 labeling was observed at the basal PM, though the staining was weak (Figures 2(d), 4(a), and 5(c)). To determine whether the basal staining resulted from GLUT-4 antibody penetration through the PM into the cytoplasm, we costained cells with di-8-ANEPPS, a membrane-impermeable dye that labels the phospholipid bilayer (Figure 4(b)).

Di-8-ANEPPS labeled the PM as well as other cell membranous structures, including the SSC, and overlapped with GLUT-4 labeling at the basolateral wall (Figures 4(a)-4(c)). To clarify the localization of GLUT-4, we colabelled cells using anti-prestin antibodies. Prestin was expressed only in OHCs at the outermost layer of the PM along the lateral wall. High-magnification images (inset in Figure 5(b)) revealed that the two proteins did not colocalize; prestin was confined to the outermost PM layer, while GLUT-4 was just inside, likely at the SSC.

We next examined the effect of receptor P2X7 agonists on the uptake of 2-NBDG (Figure 6; all data are relative to untreated cells). Intracochlear ATP is an important mediator in the regulation of hearing, and it affects the transmembrane potential in OHCs $[19,20]$. The ATP receptor P2X7 is expressed on the lateral wall of OHCs $[10,11]$, similar to the distribution of GLUT-4 (data below). Thus, we examined the effect of ATP on 2-NBDG uptake by OHCs. ATP increased the uptake of 2-NBDG significantly to $1.7582 \pm 0.5625$. 


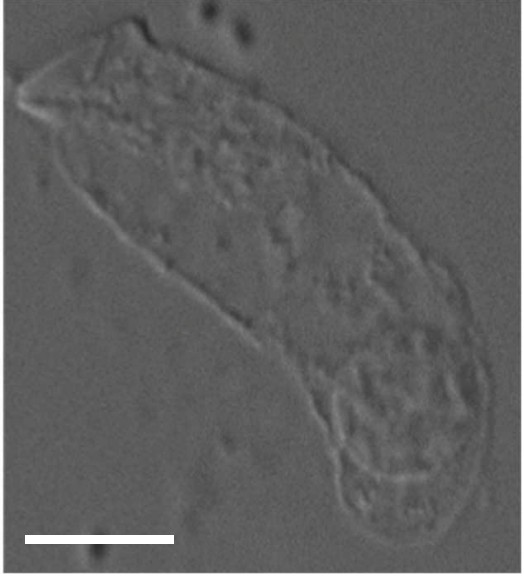

(a)

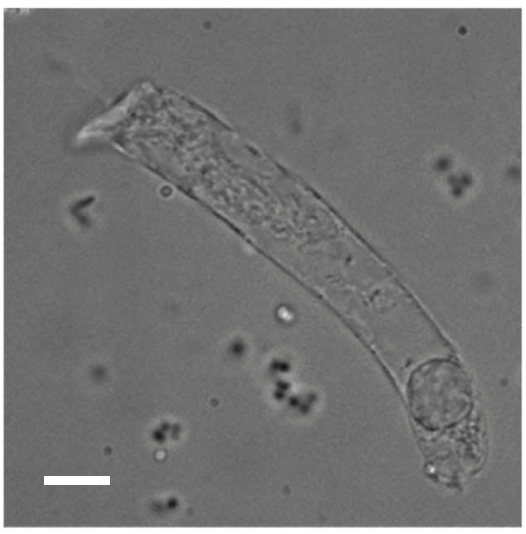

(c)

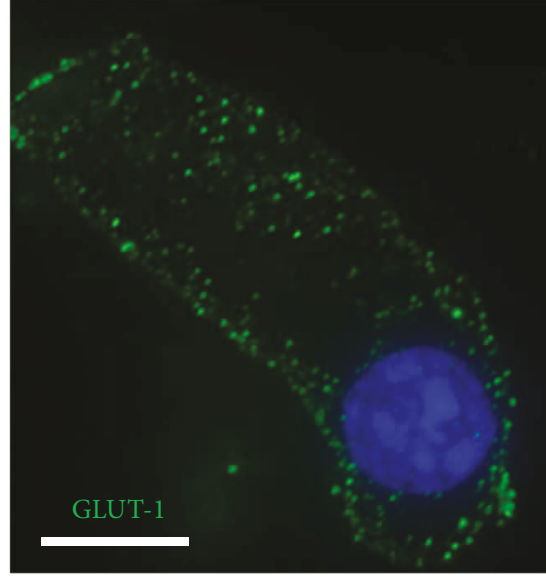

(b)

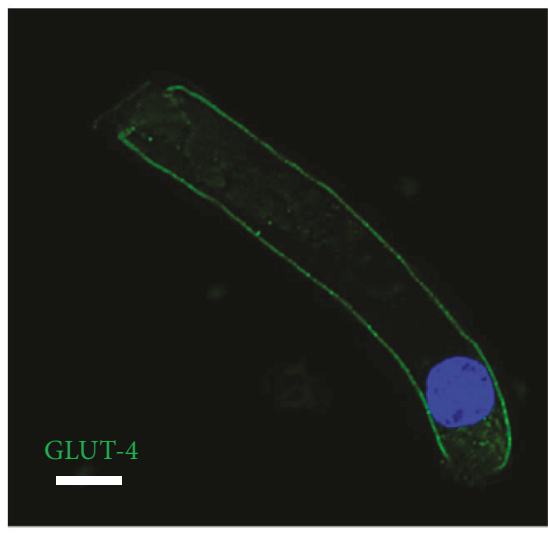

(d)

FIgURE 2: Subcellular distribution of GLUT isoforms in OHCs. Deconvoluted images of OHCs labeled with antibodies specific for various glucose transporters (green). (b) GLUT-1 and (d) GLUT-4. Blue staining corresponds to Hoechst 33342 (nuclei). (a) and (c) are Nomarski images. Scale bar, $10 \mu \mathrm{m}$.

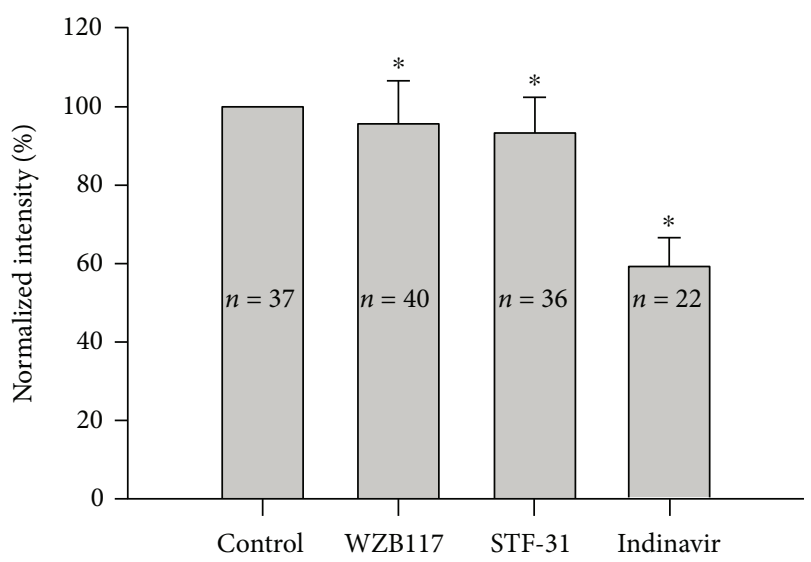

FIGURE 3: The antagonists of GLUT-1 and GLUT-4 alter the uptake of 2-NBDG. WZB117 and STF-31 are GLUT-1 antagonists and indinavir is a GLUT-4 antagonist. Error bars represent the standard error. $*$ indicates $p<0.05$ by a paired $t$-test.

\section{Discussion}

In this study, we demonstrated the uptake of 2-NBDG by OHCs. We further demonstrated that the uptake was timeand concentration-dependent and regulated by ATP receptors and glucose transporters.

Our examination of the distribution of GLUT isoforms using specific antibodies identified the expression of glucose transporters on the cuticular plate, in accordance with our live-cell imaging results showing glucose uptake at this location (data not shown). GLUT-1 expressed on all parts of OHCs scatteredly; it was not only in the cytoplasma but also on the cell membranes. The GLUT-1 inhibitors STF-31 and WZB117 $[15,21]$ inhibited the uptake of 2-NBDG, indicating that glucose are transported through GLUT-1. There is no study showing the GLUT expression in the OHCs; only some studies elucidate GLUT expression in the stria vascularis and the spiral ligament. Takeuchi and Ando [22] and Ando et al. [23] showed that GLUT-1 was expressed in the stria vascularis, whereas in basal cells and not marginal cells, the studies indicated that GLUT-1 might contribute to the transcellular glucose takeup pathway. Edamatsu et al. [14] showed that GLUT-1, GLUT-3, GLUT-5, GLUT-8, GLUT- 

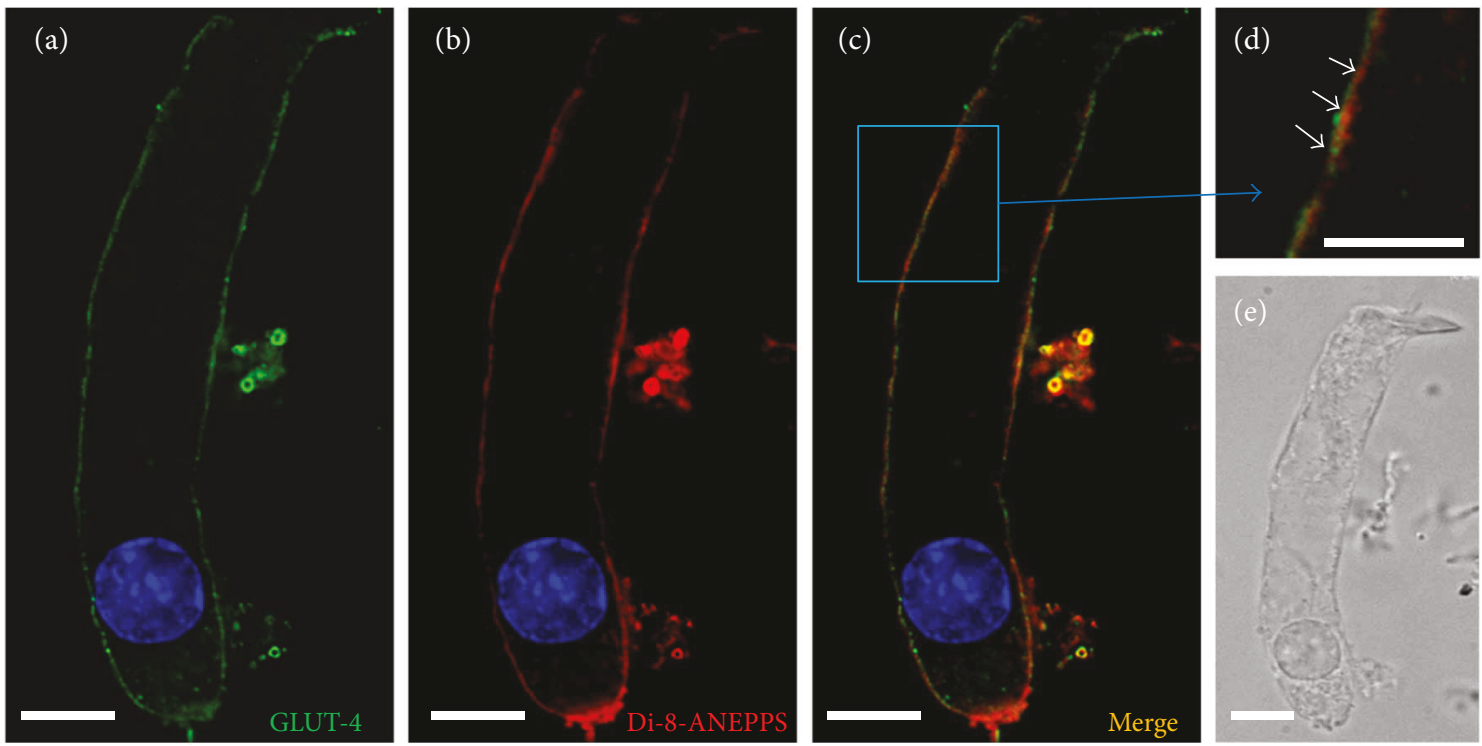

FIgURE 4: Basal staining for GLUT-4 may result from intracellular penetration of the antibody. OHCs were costained using anti-GLUT-4 antibodies and di-8-ANEPPS: (a) GLUT-4 staining; (b) di-8-ANEPPS staining; (c) merged image; (d) high magnification; (e) Nomarski image. Scale bar, $10 \mu \mathrm{m}$ in all images except the inset $(2 \mu \mathrm{m})$.

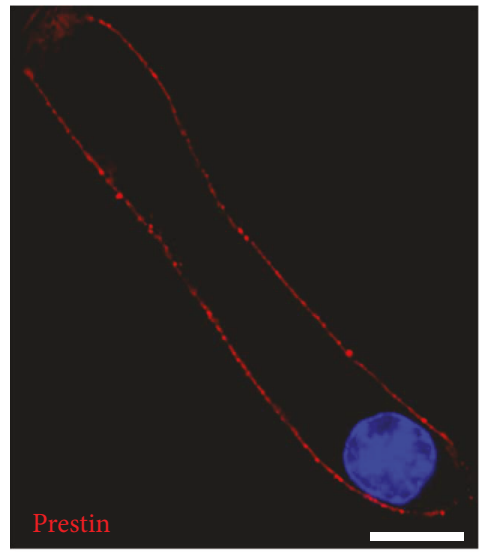

(a)

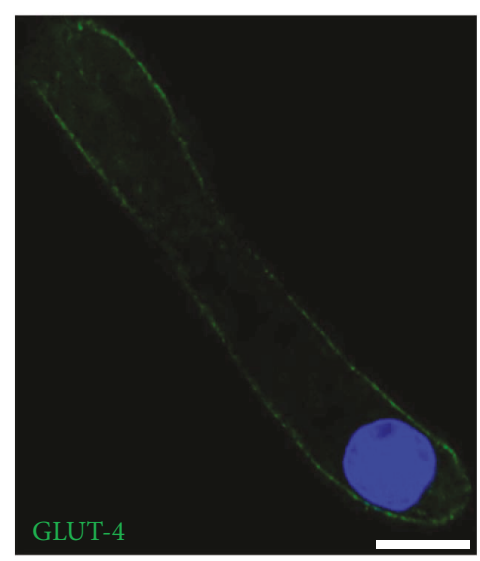

(c)

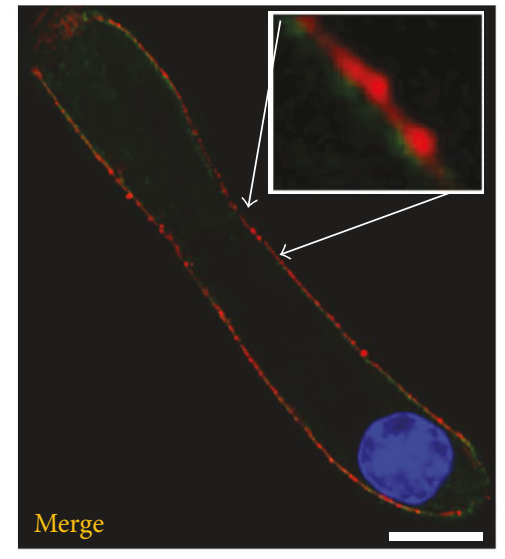

(b)

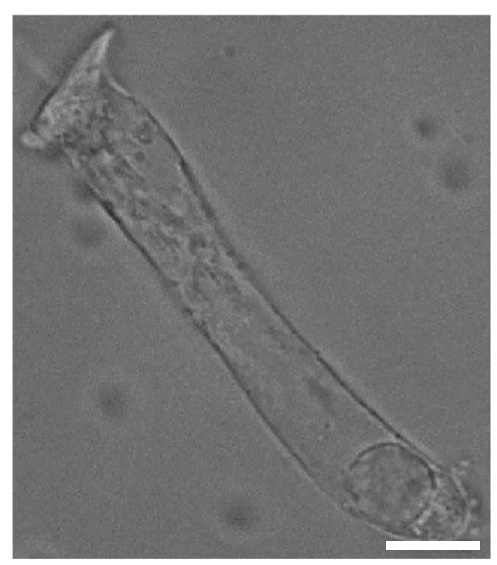

(d)

FIgURe 5: GLUT-4 colocalizes with prestin in the lateral, but not the basal, wall of OHCs. OHCs were costained for GLUT-4 and prestin. (a) Prestin and (b) merged image. Inset: high-magnification image revealing the separation of GLUT-4 and prestin. (c) GLUT-4 and (d) Nomarski image in the same field. Scale bar, $10 \mu \mathrm{m}$ in all images except the inset $(2 \mu \mathrm{m})$. 


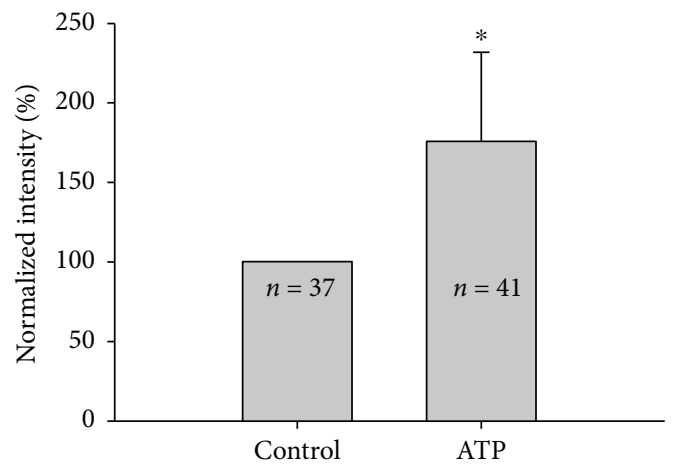

FIGURE 6: P2X7 receptor agonist alters the uptake of 2-NBDG. ATP is a P2X7 receptor agonist that could increase the uptake of 2-NBDG obviously. Error bars represent the standard error. * indicates $p<0.05$ by a paired $t$-test.

10 , and GLUT-12 were detected in both the stria vascularis and the spiral ligament and revealed that there were significant differences between the stria vascularis and the spiral ligament of GLUT-1, GLUT-4, GLUT-5, and GLUT-10 isoforms, which indicated GLUT-1, GLUT-4, GLUT-5, and GLUT-10 isoforms involved in glucose transport in the stria vascularis and the spiral ligament.

In this study, we observed that the outer hair cells expressed GLUT-5 scatteredly (data was not shown), which was consistent to the results of Belyantseva et al. [24]. They found that GLUT-5 and prestin were highly expressed in the lateral membrane of OHCs, indicating that GLUT-5 might be involved in the control of cochlear electromotility, whereas the results were different from $\mathrm{Wu}$ et al. [9] which showed that GLUT-5 was not detected in the OHCs and GLUT-5 was not necessary for OHC motility or cochlear amplification. The discrepancy may due to the different types of experimental animals. Our observation of GLUT-4 expression along the entire lateral wall of OHCs suggests that it is responsible for most glucose transport in these cells. Then we used the GLUT-4 antagonist indinavir to exam the function of GLUT-4 and we observed that the uptake of 2-NBDG decreased when the indinavir was applied. Through these results, we can suggest that GLUT-4 mainly relates with glucose transport in OHCs.

At the same time, we observed that GLUT- 4 was localized to a layer just inside the membrane where prestin was expressed, namely, the inner SSC of the OHC lateral wall [7]. Ning et al. [25] showed that the distribution of the P2X7 receptor overlapped with the prestin expression. Thus, the subcellular distribution of GLUT-4 in OHCs also resembled that of P2X7. Prestin is a unique ATP- and $\mathrm{Ca}^{2+}$-independent motor protein of the outer hair cell, involved in the electromotility and cochlear amplification [26-28]. The process needs a lot of energy consumption provided by ATP, which is also the P2X7 receptor agonist $[29,30]$. This study showed that the uptake of 2-NBDG increased after the application of ATP. ATP enhanced glucose uptake, suggesting that GLUT-4 and P2X7 may participate in electromotility. The distribution of GLUT-4 also suggests that it is related to electromotility; however, more evidence is needed to verify this hypothesis.
Diabetes is a known risk reason for hearing loss, and some previous studies suggest that GLUT-4 mutations are linked to diabetes. GLUT-4 plays an important role in the pathophysiology of T2DM, and its defective expression will hinder the entrance of glucose into the cell for energy production [31-33]. Altered insulin sensitivity may influence the expression of GLUT-4, leading to the death of OHCs because of glucose deficiency. Our observation that GLUT4 was the most strongly expressed GLUT isoform in OHCs may explain why diabetes can cause deafness. Degerman et al. [34] found that the sensory epithelium of human saccule expressed insulin-sensitive glucose transporter (GLUT4), which also exhibited expression of the insulin receptor, insulin receptor substrate 1 , and protein kinase $B$, indicating that those proteins might have a role in mechanism between diabetes and hearing loss. Thus, GLUT-4 may participate in the mechanism of hearing impairment of diabetes.

In summary, glucose is transported into OHCs across the lateral membrane via glucose transporters 1 and 4 mainly, and this transport is regulated by ATP and glucose antagonists. GLUT-4 is expressed on the inner OHC lateral walls, suggesting that it is related to electromotility. In conclusion, the lateral walls of cochlear OHCs are involved in controlling energy transport.

\section{Conflicts of Interest}

The authors declare that they have no conflicts of interest.

\section{Authors' Contributions}

Xiao-ting Cheng and Feng-bo Yang contributed equally to this work.

\section{Acknowledgments}

This project was supported by the National Basic Research Program of China (973 Program) (2014CB943002), the National Natural Science Foundation of China (nos. 81470700 and 81528005, http://www.nsfc.gov.cn), and Noise Grant (BWS14J045). The authors thank Ms. Can Xie and Dr. Hai-long Wang for the technical support. The English in this document has been checked by Dr. Anping Xia and at least two professional editors, both native speakers of English. For a certificate, please see http://www.textcheck. com/certificate/b5FiuP.

\section{References}

[1] B. Kachar, W. E. Brownell, R. Altschuler, and J. Fex, "Electrokinetic shape changes of cochlear outer hair cells," Nature, vol. 322, no. 6077, pp. 365-368, 1986.

[2] J. Ashmore, "Cochlear outer hair cell motility," Physiological Reviews, vol. 88, no. 1, pp. 173-210, 2008.

[3] S. Ramamoorthy and A. L. Nuttall, "Outer hair cell somatic electromotility in vivo and power transfer to the organ of Corti," Biophysical Journal, vol. 102, no. 3, pp. 388-398, 2012.

[4] Y. Zhu, C. Liang, J. Chen, L. Zong, G. D. Chen, and H. B. Zhao, "Active cochlear amplification is dependent on supporting cell gap junctions," Nature Communications, vol. 4, p. 1786, 2013. 
[5] K. Saito, "Fine structure of the sensory epithelium of guineapig organ of Corti: subsurface cisternae and lamellar bodies in the outer hair cells," Cell and Tissue Research, vol. 229, no. 3, pp. 467-481, 1983.

[6] L. Song and J. Santos-Sacchi, "An electrical inspection of the subsurface cisternae of the outer hair cell," Biophysical Journal, vol. 108, no. 3, pp. 568-577, 2015.

[7] N. Yu, M. L. Zhu, and H. B. Zhao, "Prestin is expressed on the whole outer hair cell basolateral surface," Brain Research, vol. 1095, no. 1, pp. 51-58, 2006.

[8] X. Wu, B. Currall, T. Yamashita, L. L. Parker, R. Hallworth, and J. Zuo, "Prestin-prestin and prestin-GLUT5 interactions in HEK293T cells," Developmental Neurobiology, vol. 67, no. 4, pp. 483-497, 2007.

[9] X. D. Wu, X. Wang, J. G. Gao et al., "Glucose transporter 5 is undetectable in outer hair cells and does not contribute to cochlear amplification," Brain Research, vol. 1210, pp. 20-28, 2008.

[10] H. B. Zhao, N. Yu, and C. R. Fleming, "Gap junctional hemichannel-mediated ATP release and hearing controls in the inner ear," Proceedings of the National Academy of Sciences of the United States of America, vol. 102, no. 51, pp. 1872418729, 2005.

[11] N. Yu and H. B. Zhao, "ATP activates P2x receptors and requires extracellular $\mathrm{Ca}^{++}$participation to modify outer hair cell nonlinear capacitance," Pflügers Archiv - European Journal of Physiology, vol. 457, no. 2, pp. 453-461, 2008.

[12] K. Yoshioka, H. Takahashi, T. Homma et al., "A novel fluorescent derivative of glucose applicable to the assessment of glucose uptake activity of Escherichia coli," Biochimica et Biophysica Acta (BBA) - General Subjects, vol. 1289, no. 1, pp. 5-9, 1996.

[13] Z. Su, "Techniques for isolating hair cells from guinea pig cochlea," Zhonghua Er Bi Yan Hou Ke Za Zhi, vol. 27, no. 3, pp. 133-135, 1992.

[14] M. Edamatsu, Y. Kondo, and M. Ando, "Multiple expression of glucose transporters in the lateral wall of the cochlear duct studied by quantitative real-time PCR assay," Neuroscience Letters, vol. 490, no. 1, pp. 72-77, 2011.

[15] D. A. Chan, P. D. Sutphin, P. Nguyen et al., "Targeting GLUT1 and the Warburg effect in renal cell carcinoma by chemical synthetic lethality," Science Translational Medicine, vol. 3, no. 94, article 94ra70, 2011.

[16] K. R. Boheler, S. Bhattacharya, E. M. Kropp et al., "A human pluripotent stem cell surface $\mathrm{N}$-glycoproteome resource reveals markers, extracellular epitopes, and drug targets," Stem Cell Reports, vol. 3, no. 1, pp. 185-203, 2014.

[17] I. Igbe, E. K. Omogbai, and A. O. Oyekan, "Renal effects of glucose transporter 4 in $\mathrm{N} \omega$-nitro-L-arginine/ /high salt-induced hypertensive rats," Bratislava Medical Journal, vol. 116, no. 1, pp. 57-63, 2015.

[18] C. Wilson, A. Contreras-Ferrat, N. Venegas et al., "Testosterone increases GLUT4-dependent glucose uptake in cardiomyocytes," Journal of Cellular Physiology, vol. 228, no. 12, pp. 2399-2407, 2013.

[19] S. G. Kujawa, C. Erostegui, M. Fallon, J. Crist, and R. P. Bobbin, "Effects of adenosine $5^{\prime}$-triphosphate and related agonists on cochlear function," Hearing Research, vol. 76, no. 1-2, pp. 87-100, 1994.

[20] D. J. B. Muñoz, P. R. Thorne, G. D. Housley, T. E. Billett, and J. M. Battersby, "Extracellular adenosine 5 '-triphosphate
(ATP) in the endolymphatic compartment influences cochlear function," Hearing Research, vol. 90, no. 1-2, pp. 106-118, 1995.

[21] Y. Liu, Y. Cao, W. Zhang et al., "A small-molecule inhibitor of glucose transporter 1 downregulates glycolysis, induces cell-cycle arrest, and inhibits cancer cell growth in vitro and in vivo," Molecular Cancer Therapeutics, vol. 11, no. 8, pp. 1672-1682, 2012.

[22] S. Takeuchi and M. Ando, "Marginal cells of the stria vascularis of erbils take up glucose via the facilitated transporter GLUT: application of autofluorescence," Hearing Research, vol. 114, no. 1-2, pp. 69-74, 1997.

[23] M. Ando, M. Edamatsu, S. Fukuizumi, and S. Takeuchi, "Cellular localization of facilitated glucose transporter 1 (GLUT-1) in the cochlear stria vascularis: its possible contribution to the transcellular glucose pathway," Cell and Tissue Research, vol. 331, no. 3, pp. 763-769, 2008.

[24] I. A. Belyantseva, H. J. Adler, R. Curi, G. I. Frolenkov, and B. Kachar, "Expression and localization of prestin and the sugar transporter GLUT-5 during development of electromotility in cochlear outer hair cells," The Journal of Neuroscience, vol. 20, no. 24, article RC116, 2000.

[25] Y. Ning, Z. Hong-bo, Y. Shi-ming, and Z. Suo-qiang, "Distribution of $\mathrm{P} 2$ receptors on outer hair cell membrane," Journal of Otology, vol. 6, pp. 441-441, 2008.

[26] J. Zheng, W. Shen, D. Z. Z. He, K. B. Long, L. D. Madison, and P. Dallos, "Prestin is the motor protein of cochlear outer hair cells," Nature, vol. 405, no. 6783, pp. 149-155, 2000.

[27] M. C. Liberman, J. G. Gao, D. Z. Z. He, X. D. Wu, S. P. Jia, and J. Zuo, "Prestin is required for electromotility of the outer hair cell and for the cochlear amplifier," Nature, vol. 419, no. 6904, pp. 300-304, 2002.

[28] P. Dallos and B. Fakler, "Prestin, a new type of motor protein," Nature Reviews Molecular Cell Biology, vol. 3, no. 2, pp. 104111, 2002.

[29] M. Kukley, P. Stausberg, G. Adelmann, I. P. Chessell, and D. Dietrich, "Ecto-nucleotidases and nucleoside transporters mediate activation of adenosine receptors on hippocampal mossy fibers by $\mathrm{P} 2 \mathrm{X}_{7}$ receptor agonist $2^{\prime}-3^{\prime}$-O-(4-benzoylbenzoyl)-ATP," The Journal of Neuroscience, vol. 24, no. 32, pp. 7128-7139, 2004.

[30] S. F. Moore and A. B. Mackenzie, "Species and agonist dependent zinc modulation of endogenous and recombinant ATPgated $\mathrm{P}_{2} \mathrm{X}_{7}$ receptors," Biochemical Pharmacology, vol. 76, no. 12, pp. 1740-1747, 2008.

[31] M. Sato, N. Dehvari, A. I. Oberg et al., "Improving type 2 diabetes through a distinct adrenergic signaling pathway involving mTORC2 that mediates glucose uptake in skeletal muscle," Diabetes, vol. 63, no. 12, pp. 4115-4129, 2014.

[32] J. S. Hansen, X. Zhao, M. Irmler et al., "Type 2 diabetes alters metabolic and transcriptional signatures of glucose and amino acid metabolism during exercise and recovery," Diabetologia, vol. 58, no. 8, pp. 1845-1854, 2015.

[33] S. T. Yan, C. L. Li, H. Tian et al., "MiR-199a is overexpressed in plasma of type 2 diabetes patients which contributes to type 2 diabetes by targeting GLUT4," Molecular and Cellular Biochemistry, vol. 397, no. 1-2, pp. 45-51, 2014.

[34] E. Degerman, U. Rauch, S. Lindberg, P. Caye-Thomasen, A. Hultgårdh, and M. Magnusson, "Expression of insulin signalling components in the sensory epithelium of the human saccule," Cell and Tissue Research, vol. 352, no. 3, pp. 469$478,2013$. 


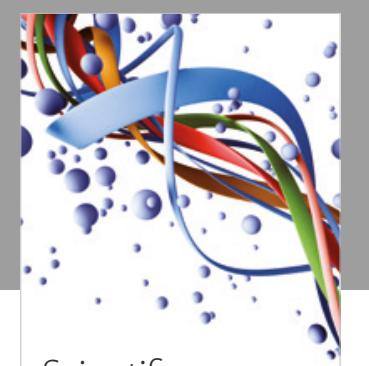

Scientifica
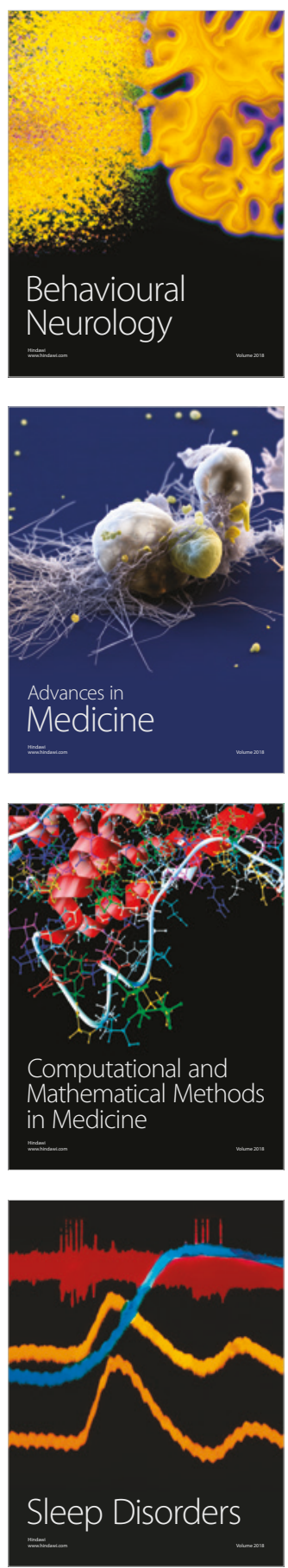

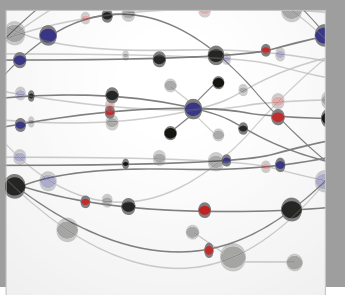

The Scientific World Journal

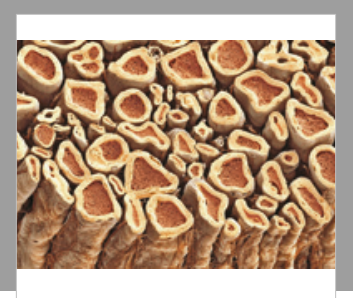

Case Reports in

Neurological Medicine

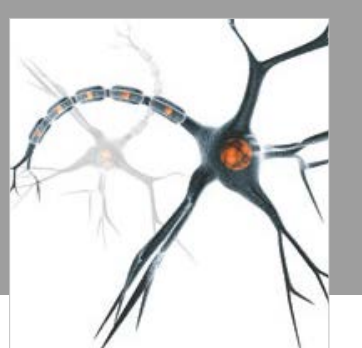

Neural Plasticity

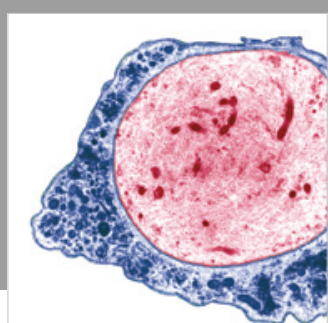

Multiple Sclerosis

International

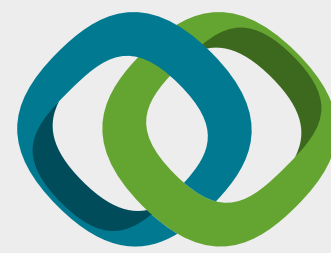

Hindawi

Submit your manuscripts at

www.hindawi.com
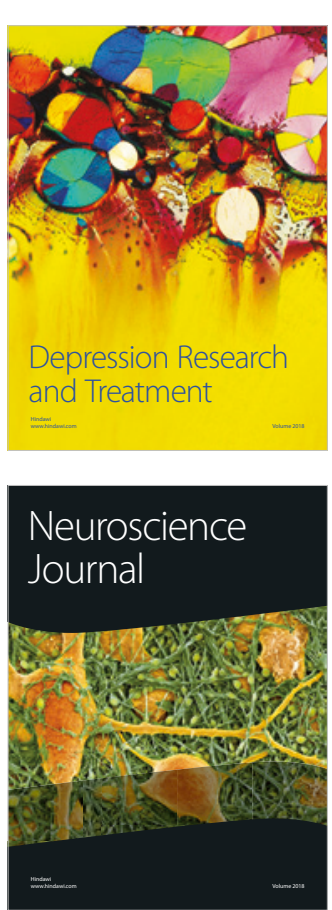

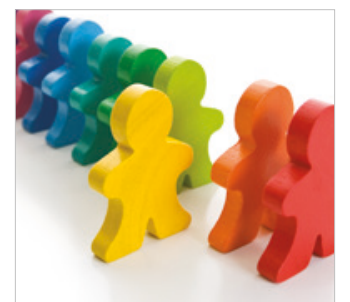

Autism

Research and Treatment
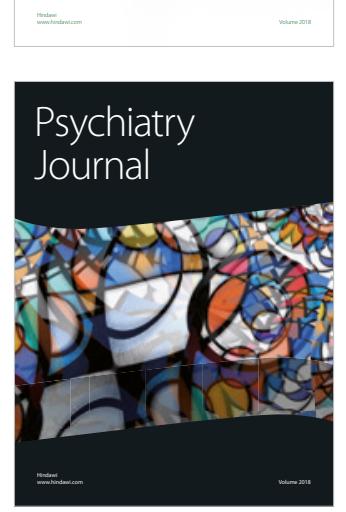
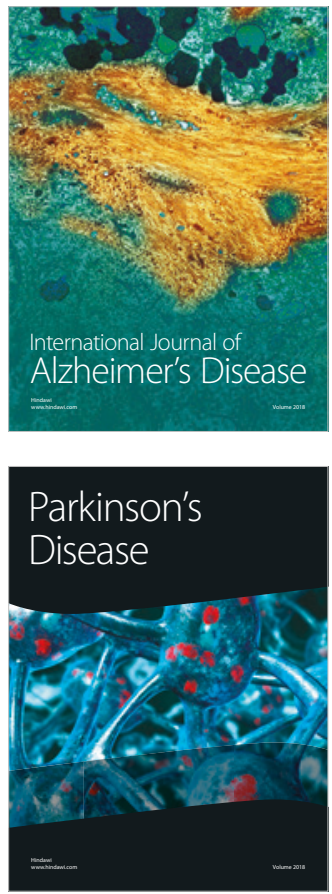
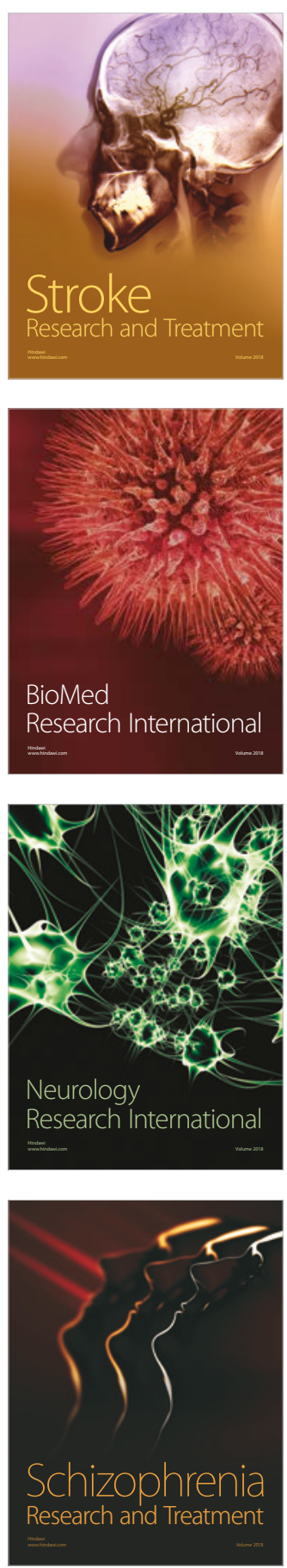